\title{
The first investigation on differences in the effectiveness of mycophenolate mofetil and azathioprine antimetabolites determined in Polish patients treated for non-infectious uveitis
}

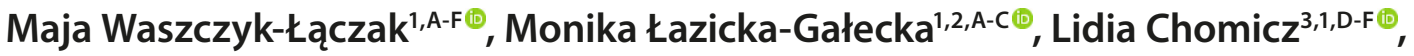 \\ Krzysztof Mucha ${ }^{4, F}{ }^{\oplus}$, Leszek Pączek ${ }^{4, F}{ }^{\infty}$, Jacek P. Szaflik ${ }^{1,2, A, E-F} \oplus$ \\ 1 SPKSO Ophthalmic Hospital, Warsaw, Poland \\ 2 Department of Ophthalmology, Medical University, Warsaw, Poland \\ ${ }^{3}$ Department of Medical Biology, Medical University, Warsaw, Poland \\ ${ }^{4}$ Department of Immunology, Transplantology, and Internal Diseases, Medical University, Warsaw, Poland \\ A - Research concept and design, B - Collection and/or assembly of data, C - Data analysis and interpretation, \\ $D$ - Writing the article, $E$ - Critical revision of the article, $F$ - Final approval of article
}

\begin{abstract}
Waszczyk-Łączak M, Łazicka-Gałecka M, Chomicz L, Mucha K, Pączek L, Szaflik JP. The first investigation on differences in the effectiveness of mycophenolate mofetil and azathioprine antimetabolites determined in Polish patients treated for non-infectious uveitis. Ann Agric Environ Med. 2020; 27(4): 644-649. doi: 10.26444/aaem/125837
\end{abstract}

\begin{abstract}
I Abstract
Introduction. The non-infectious uveitis, a serious vision-threatening disease is the fourth most common cause of blindness in working population of the developed world. Various antimetabolites are applied in corticosteroid-sparing therapy also in Poland but their efficacy was not compared in our country. The aim of our study was to compare mycophenolate mofetil and azathioprine in terms of therapeutic effect of the antimetabolites in Polish patients with this disease.

Materials and method. The comparative, retrospective study included data of 61 patients admitted to Independent Public University Eye Hospital between January 2009 and January 2017, treated with antimetabolites for non-infectious uveitis. 31 patients received mycophenolate mofetil, 30 patients - azathioprine. In the assessment of corticosteroid-sparing efficacy, among others changes in visual acuity, the duration of the disease and therapy, incidence of ophthalmologic complications, adverse systemic side effects were determined.

Results. The corticosteroid-sparing therapy was more often effective, and an improvement of visual acuity more frequent in patients treated with mycophenolate mofetil than in these receiving azathioprine ( $84 \%$ patients vs. $60 \%$, and $27 \%$ patients vs. $13 \%$, respectively); these differences were statistically significant $(p<0.05)$.

Conclusions. Results of our study showing better therapeutic efficacy when applied mycophenolate mofetil seems promising approach for treatment of non-infectious posterior uveitis and panuveitis. In the first study, there was different duration of the disease before drug administration (10.5 years vs. 7.14 years in the azathioprine and mycophenolate mofetil therapy, respectively, $\mathrm{p}<0.05$ ) and limited number of patients assessed, thus it is desirable to examine more Polish patients treated with the antimetabolites.
\end{abstract}

\section{Keywords}

non-infectious uveitis, immunosuppression, mycophenolate mofetil, azathioprine, Polish patients, therapeutic effectiveness

\section{INTRODUCTION}

Chronic non-infectious uveitis is a rare but vision-threatening disease that particularly affects young and middle-aged persons; the illness is the fourth most common cause of blindness in the working-age population of the developed world [1]. Non-infectious uveitis is a heterogeneous group of diseases in which the common denominator is the presence of sight-threatening inflammation within the structures of the eye. The inflammation can be a manifestation of some systemic autoimmune disease such as one of the spondyloarthritides, Behcet's disease, Vogt-KoyanagiHarada (VKH) syndrome, systemic lupus erythematosus, sarcoidosis, autoimmune hepatitis, and multiple sclerosis [2-10] or a condition affecting only the eyeball. More often,

Address for correspondence: Lidia Chomicz, Department of Medical Biology, Medical University of Warsaw, Litewska 14/16, 00-575, Warsaw, Poland E-mail: lidia.chomicz@wum.edu.pl

Received: 29.03.2020; accepted: 29.07.2020; first published: 03.09.2020 however, the cause remains undefined and is classified as an idiopathic [11].

Biziorek et al. [12] presented the results of a prospective study of 563 patients with uveitis in whom the diagnosis was not established in $30.0 \%$ of all cases; moreover, among patients with intermediate uveitis even $73.2 \%$ were without causative diagnosis. In several current studies on an inflammation of uveal tissues in Korean and Iranian populations [13, 14], the relationships between uveitis and ocular toxocarosis were demonstrated, although, in some analyzed cases of the zoonotic parasitosis the cause of uveitis could not be determined. For this reason, among the idiopathic cases of uveitis, particularly in those with posterior uveitis, a diagnosis to exclude toxocarosis should be considered.

The clinical picture of chronic uveitis depends on the location, severity and the duration of the inflammatory process, which affects the development and variety of symptoms and results in diagnostic and therapeutic difficulties. The success of the uveitis therapy depends greatly 
on the prompt beginning of treatment. The prolongation of the inflammatory process that covers greater areas of healthy tissue may result in irreversible deterioration of vision, or even complete blindness. To control the inflammation, the first-line therapy for chronic uveitis is oral prednisone [15, 16]. However, for some patients, systemic corticosteroids are insufficient to control the disease and then the corticosteroidsparing protocols are used.

Guidelines for the Use of Immunosuppressive Drugs in Patients With Ocular Inflammatory Disorders published in 2000 [17] provide recommendations for the use of corticosteroid-sparing therapy in the following cases:

- the inability to control inflammation after one month of high-dose corticosteroid treatment;

- the inability to maintain control of ocular inflammation with prednisolone dose $\leq 10 \mathrm{mg}$ daily,

- the presence of corticosteroid-related side effects that require further tapering or discontinuation of corticosteroid application;

- ocular inflammatory diseases known to have a poor prognosis with corticosteroid treatment alone.

There are four groups of corticosteroid-sparing agents: antimetabolites, T-cell inhibitors, alkylating agents and biologic response modifiers (BRM). Currently, antimetabolites are the most common first-line corticosteroid-sparing therapy used in chronic non-infectious uveitis. Different antimetabolites are applied in corticosteroid- sparing therapy also in Poland but their effectiveness was not compared in Polish patients with this disease. This class of drugs include mycophenolate mofetil (MMF), azathioprine (AZA) and methotrexate (MTX). As we know, antimetabolites inhibit the synthesis of nucleotides.

Mycophenolate mofetil (MMF) is a prodrug and after absorption in the gastrointestinal tract, in the liver it undergoes transformation into mycophenolic acid (MPA). The latter is a selective, reversible and non-competitive inhibitor of inosine monophosphate dehydrogenase (IMPDH), enzyme that is involved in the synthesis of guanine nucleosides. The immediate consequence of inhibiting its activity is DNA synthesis blocking, as well as reducing GTP (Guanosine5'triphosphate) resources and impairing the glycosylation of proteins.

There are two ways to obtain guanosine in most of the body cells. Either with the IMPDH or with the so-called "recovery route” of purine degradation products. In T- and B-lymphocytes, guanosine is formed mainly via the first path, while in the other cells the recovery route predominates. Therefore, MMF is a selective cytostatic against both, the T- and B-lymphocytes [18].

Azathioprine (AZA) is a purine nucleoside analogue, more specifically a heterocyclic derivative of 6-mercaptopurine. The anti-metabolism effect of AZA is associated with its incorporation into the DNA chain. As a result, the biosynthesis of nucleic acids is inhibited and, consequently, the cells that are involved in inflammation do not undergo proliferation. Immunologically, AZA e.g.: decreases the number of peripheral T- and B-lymphocytes, and reduces mixed lymphocyte reactivity, interleukin-2 synthesis and IgM production. The onset of the therapeutic effect occurs after a few weeks or even months after the beginning of the AZA therapy because of its dynamics [19].

\section{OBJECTIVE}

The aim of this research was to compare the mycophenolate mofetil and azathioprine in terms of possible differences in therapeutic effectiveness of the antimetabolites in Polish patients with non-infectious uveitis.

\section{MATERIALS AND METHOD}

We conducted analysis of data from patients with noninfectious uveitis, admitted to Independent Public University Eye Hospital in Warsaw from January 2009 to January 2017. The retrospective and non-randomised study was performed in accordance with the tenets of the Declaration of Helsinki and approved by the local ethics committee.

The evaluation included 61 patients with non-infectious uveitis treated with antimetabolites who received immunosuppression (IS). The exclusion criteria were: patients with the follow-up period shorter than 3 months and patients with an inflammation affecting tissues other than the uvea.

Our study comprised two groups of patients: 31 patients of Group 1 received mycophenolate mofetil (MMF), and 30 patients of Group 2 received azathioprine (AZA). Both groups were dominated by women: Group $1-24$ patients (77.4\%) and Group 2 - 23 patients (76.7\%). Patients treated with MMF were between 18 and 76 years of age, the average age was 42.9 years; patients treated with AZA were between 22 and 70 years of age, the average age was 48.5 years; the difference in the average age of patients from both groups was not statistically significant $(\mathrm{p}=0.19)$.

Clinical data also included the diagnosis of kind uveitis, uni/bilaterality of uveitis, ocular complications, surgical interventions, duration of the disease before therapy, indication for antimetabolite therapy, antimetabolite dosage variables, duration of the therapy, corticosteroid-sparing success, changes in visual acuity and adverse systemic side effects. Anterior uveitis and panuveitis were prevalent in both groups; no statistical differences were revealed as regards the laterality of uveitis or the uveal segment that was affected by the inflammatory process in the examined groups.

The immunosuppression (IS) treatment was determined individually for each patient; the dose depended on the type of uveitis and general health of the patient comprising the body weight and concomitant systemic diseases.

The majority of patients that received MMF were initially administered $1000 \mathrm{mg}$ twice daily (61\%) or $750 \mathrm{mg}$ twice daily (29\%).

In the AZA group, similar regimen was implemented. The treatment was started with a possibly highest dose, i.e. $150 \mathrm{mg}$ twice daily (33\%) or $100 \mathrm{mg}$ twice daily (30\%). At the beginning of the therapy, six patients from this group were treated with MMF for one month, and other three patients continued the treatment for 3 months. As AZA therapy in those nine patients was disproportionately longer compared to the above-mentioned MMF periods, those patients have been included in the AZA group.

After obtaining a 3-month period of the stabilization of the local status, the dose was gradually reduced with the aim of drug discontinuation. If uveitis recurred during the dose reduction process, the dose of the basic immunosuppressive drug was increased again. 
Due to the risk of viral co-infections (e.g. Herpes Simplex Virus, Cytomegalovirus, Human Papillomavirus) and atypical infections (Pneumocystis jiroveci), which may occur during immunosuppressive therapy, 6-month prophylaxis with acyclovir $(4 \times 400 \mathrm{mg}$ daily) and co-trimazole $(480 \mathrm{mg}$ twice daily for 3 months tapered to $480 \mathrm{mg}$ daily) was introduced. [20] Possible systemic side effects (e.g. gastrointestinal, haematological) and the pre-dose MPA plasma concentration was measured each time when the MMF dosage was changed.

Uveitis subtype classification was based on the recommendations of the Standardization of Uveitis Nomenclature (SUN) Working Group [21]. Complete ophthalmological examination, including best-corrected visual acuity (BCVA), tonometry, biomicroscopy and indirect ophthalmoscopy were carried out on all patients. Additionally, optical coherence tomography (OCT) or fluorescein angiography were performed in patients with Cystoid Macular Oedema (CMO). Ultrasound was used when the inflammation affected the vitreous. BCVA was measured with the Snellen's Chart and converted to logMAR using a standard formula. Because of limitations of this chart we assumed that $\log M A R$ values of 1.9 meant counting fingers, 2.2 - hand movements, 2.7 - light perception and 3.0 - no light perception. We created three groups of patients to compare changes in visual acuity. In group A, we recorded the lowest values of visual acuity $\geq 1.0$, in group $B$ - intermediate values, in group $\mathrm{C} \leq 0.3$.

The control of the inflammation was defined as an inactive disease at a prednisolone dose of $\leq 10 \mathrm{mg}$ daily (or an equivalent of another steroid e.g. methylprednisolone) as recommended by SUN Working Group [21]. Furthermore, we decreased the dose to $5 \mathrm{mg}$ daily or discontinued corticosteroid administration. The lack of uveitis activity recorded over at least two visits scheduled at least 28 days apart, was considered to be the therapeutic success.

Data analysis. Descriptive methods and methods of statistical inference were used for the analysis of data. In order to compare the frequency of occurrence of individual varieties of features in the study groups and to investigate the relationship between variables, the chi-squared independence test was used. Fisher's exact test was used to compare the frequency. Before comparing the means in the study groups, the compatibility of the distribution of the analyzed measurable variables with the normal distribution was checked using the Shapiro-Wilk test. Since the distributions of the measurable variables analyzed differed significantly from the normal distribution, non-parametric Mann-Whitney test was used to compare the means in the groups. In case of comparisons of several sub-groups, the Kruskal-Wallis test was used, a nonparametric equivalent of the analysis of variance for a single classification. The significance of the correlation coefficient was assessed using the Student's t-test. Statistically significant differences were found between means (or frequencies) and those relations between variables for which the calculated test value was equal to or greater than the critical value read from the respective tables with the right number of degrees of freedom and the probability of error $\mathrm{p}<0.05$.

\section{RESULTS}

Characteristics of patients. The characteristics of the noninfectious uveitis in patient groups analyzed in terms of the type of inflammation is shown in Table 1 . The majority of patients had bilateral disease: $68 \%$ in Group 1 receiving mycophenolate mofetil and 57\% in patients of Group 2 receiving azathioprine.

Table 1. Characteristics of non-infectious uveitis in patient groups analyzed

\begin{tabular}{lcccc}
\hline & \multicolumn{2}{c}{ Group 1 (MMF) } & \multicolumn{2}{c}{ Group 2 (AZA) } \\
\cline { 2 - 6 } & number of patients & $\%$ & number of patients & $\%$ \\
\hline Laterality of uveitis & 21 & 67.7 & 17 & 56.7 \\
\hline Bilateral & 4 & 12.9 & 6 & 20.0 \\
\hline Right eye & 6 & 19.4 & 7 & 23.3 \\
\hline Left eye & 11 & 35.5 & 10 & 33.3 \\
\hline Type of uveitis & 5 & 16.1 & 8 & 26.7 \\
\hline Anterior & 7 & 22.6 & 2 & 6.7 \\
\hline Intermediate & 8 & 25.8 & 10 & 33.3 \\
\hline Posterior & & & & \\
\hline Panuveitis & & & & \\
\hline
\end{tabular}

Group 1 (MMF) - patients treated with mycophenolate mofetil, Group 2 (AZA) - patients treated with azathioprine.

The comparison of the duration of the uveitis before administration of antimetabolites to patients of study groups is presented in Table 2. There was different duration of the disease before the drug administration: significantly longer in the patients from Group $2-10.5$ years, than in those from Group $1-7.14$ years; the difference was statistically significant $(\mathrm{p}<0.03)$.

Table 2. Comparison of duration of uveitis before administration of antimetabolites to patients of study groups

\begin{tabular}{lcccccc}
\hline \multirow{2}{*}{ Group } & \multicolumn{6}{c}{ Calculated disease length parameters (years) } \\
\cline { 2 - 7 } & $\min$ & $\max$ & $\mathrm{x}$ & $\mathrm{Me}$ & $\mathrm{SD}$ & $\mathrm{v}(\%)$ \\
\hline Group 1 (MMF) & 0.08 & 29.0 & $\mathbf{7 . 1 4}$ & 5.0 & 7.67 & 107.4 \\
\hline Group 2 (AZA) & 0.08 & 33.0 & $\mathbf{1 0 . 5}$ & 9.5 & 7.84 & 74.6 \\
\hline
\end{tabular}

$\mathrm{x}=$ average; $\mathrm{Me}=$ median; $\mathrm{SD}=$ standard deviation; $\mathrm{v}=$ coefficient of variation; $\mathrm{z}=$ the MannWhitney test $2.265 ; p=0.024$

Corticosteroid-sparing efficacy. There was different frequency between the patient groups in achieving therapeutic success defined as an inactive disease at reducing the dose to $\leq 10 \mathrm{mg}$. During the entire period studied, the steroid-sparing effectiveness was obtained more commonly in the MMF group of patients than in the patients treated with AZA: 24 vs 18 patients, $84 \%$ vs $60 \%$, respectively; the difference was statistically significant $(\mathrm{p}<0.04)$. However, there was no statistically significant difference in the period required to reduce the steroid dose to $10 \mathrm{mg}$ between the patient groups $(p=0.15)$. In each group, most patients achieved this success during 1 to 6 months: 16 patients of Group 1 (52\%) and 13 patients of Group 2 (43\%).

We investigated the correlation between therapeutic success and factors such as: the duration of the disease before inclusion, the reason for including immunosuppression and the segment of the uvea that was affected by the disease. 
There were not unequivocal relationship between the average duration of the disease before administration of antimetabolites and therapeutic effect.

Indications for the immunosuppression (IS) therapy of patients of particular groups are presented in Table 3. In both groups, there was no statistically significant correlation between the reason for the inclusion of IS and the treatment success. The most common indication for IS treatment was the failure of glucocorticoid monotherapy (Group $1=54.8 \%$; Group $2=50 \%$ ).

Table 3. Indications for treatment with antimetabolites in particular groups of patients

\begin{tabular}{|c|c|c|c|c|}
\hline & \multicolumn{2}{|c|}{ Group 1 (MMF) } & \multicolumn{2}{|c|}{ Group 2 (AZA) } \\
\hline & $\begin{array}{l}\text { number of } \\
\text { patients }\end{array}$ & $\%$ & $\begin{array}{l}\text { number of } \\
\text { patients }\end{array}$ & $\%$ \\
\hline Failure of therapy with steroids & 17 & 54.8 & 15 & 50 \\
\hline No possibility to reduce prednisone & 3 & 9.7 & 8 & 26.7 \\
\hline $\begin{array}{l}\text { The lack of efficacy of other } \\
\text { immunosuppressants }\end{array}$ & 1 & 3.2 & 0 & 0 \\
\hline Other & 10 & 32.3 & 7 & 23.3 \\
\hline
\end{tabular}

MMF = mycophenolate mofetil; $A Z A=$ azathioprine

No significant relationship was revealed between the inflammation of the uveal segment treatment success in both groups. In Group 1 the highest success rate was noted in patients with panuveitis $(88 \%)$ and in Group 2 - in patients with intermediate uveitis (62.5\%).

Incidence of ophthalmologic complications. Data on the occurrence of ophthalmologic complications in the course of uveitis in each group are presented in Table 4. Out of the all complications, only the incidence of cataracts significantly differed between the compared groups $(\mathrm{p}<0.02)$. Apparently, the patients were significantly more often treated with AZA than MMF ( 27 vs 20 , it is $90 \%$ vs $64.5 \%$, respectively).

Table 4. Comparison of the incidence of ophthalmologic complications in the studied groups

\begin{tabular}{lcccc}
\hline & \multicolumn{2}{c}{ Group 1 (MMF) } & \multicolumn{2}{c}{ Group 2 (AZA) } \\
\cline { 2 - 6 } & $\begin{array}{c}\text { number of } \\
\text { patients }\end{array}$ & $\%$ & $\begin{array}{c}\text { number of } \\
\text { patients }\end{array}$ & $\%$ \\
\hline Cataract & $20^{*}$ & $64.5^{*}$ & $27^{*}$ & $90.0^{*}$ \\
\hline Glaucoma & 9 & 29.0 & 10 & 33.3 \\
\hline Macular oedema & 10 & 32.3 & 10 & 33.3 \\
\hline Keratopathy & 2 & 6.5 & 2 & 6.7 \\
\hline Floaters & 10 & 32.3 & 15 & 50.0 \\
\hline Posterior synechiae & 11 & 35.5 & 12 & 40.0 \\
\hline Vitreous haemorrhage & 3 & 9.7 & 1 & 3.3 \\
\hline
\end{tabular}

$\mathrm{MMF}=$ mycophenolate mofetil; $\mathrm{AZA}=$ azathioprine; ${ }^{*}$ this difference is statistically significant.

No significant differences were observed as regards the incidence of all other complications. In case of patients with diagnosed cataracts, surgery was performed in 16 patients from Group 1 (80\%) and 22 patients from Group 2 (81\%).

Patients with anterior uveitis and panuveitis dominated in both groups. Capsulotomy was performed in 6 patients from Group 1 (19\%) and in 12 patients from Group 2 (40\%). The average time for capsulotomy after cataract surgery in Group 1 was 26.7 months; in Group 2 it was 23.3 months.
Improvement of visual acuity. To compare changes in visual acuity, we created three reference groups - A, B and C.

In group $A$, we recorded the worst visual acuity $\geq 1.0$, in group $\mathrm{B}$ - intermediate and in group $\mathrm{C} \leq 0.3$. The comparison of visual acuity before treatment showed no significant differences between MMF and AZA groups. Prior to the treatment, most patients were located in reference group $\mathrm{C}$, i.e. 50\% of patients from Group 1, and 55\% from Group 2 .

The comparison of changes in best-corrected visual acuity (BCVA) before and after the treatment showed a statistically significant difference between Groups 1 and $2(\mathrm{p}<0.04)$.

In Group 2 (AZA), a deterioration of visual acuity was reported in case of 4 eyes (9\%), while in Group 1 (MMF) such a situation did not occur at all. Visual acuity deteriorated in 2 eyes due to post-inflammatory lesions in the corpus vitreum, in 1 eye - due to macular oedema, and in 1 eye - corneal decompensation. There was a significant difference in the frequency of improvement of visual acuity between Group 1 and 2: 14 eyes vs 6 eyes, $27 \%$ vs $13 \%$.

Adverse systemic side effects during therapy. Data on frequency of adverse systemic side effects are shown in Table 5. Undesirable situations occurred in 11 patients from Group $1(35.5 \%)$ and 9 patients from Group 2 (30\%); the difference was not statistically significant.

Table 5. Adverse systemic side-effects during therapy

\begin{tabular}{lcccc}
\hline & \multicolumn{2}{c}{ Group 1 (MMF) } & \multicolumn{2}{c}{ Group 2 (AZA) } \\
\cline { 2 - 6 } & $\begin{array}{c}\text { number of } \\
\text { patients }\end{array}$ & $\%$ & $\begin{array}{c}\text { number of } \\
\text { patients }\end{array}$ & $\%$ \\
\hline arterial hypertension & 2 & 6.5 & 2 & 6.7 \\
\hline elevated liver enzyme levels & 1 & 3.2 & 2 & 6.7 \\
\hline hypokalaemia & 1 & 3.2 & - & - \\
\hline bone marrow suppresion & 4 & 12.9 & 3 & 10.0 \\
\hline neuropathy & - & - & 1 & 3.3 \\
\hline gastrointestinal upset & 2 & 6.5 & 4 & 13.3 \\
\hline hair loss & 2 & 6.5 & - & - \\
\hline infections & 2 & 6.5 & - & - \\
\hline death & - & - & 1 & 3.3 \\
\hline all & 11 & 35.5 & 9 & 30.0 \\
\hline
\end{tabular}

MMF = mycophenolate mofetil; AZA = azathioprine. The differences between the groups were not statistically significant.

In the MMF group, patients who experienced adverse effects were subject to longer treatment - 24.6 months, than patients who did not experience adverse effects -22.6 months. In the AZA group, the average treatment time of patients who experienced adverse effects were subject 20.9 months in comparison to those without adverse effects 18.5 months.

Because of adverse effects in 1 patient in Group 1 and in 4 patients in Group 2 the drug was discontinued.

\section{DISCUSSION}

In our investigation, we wanted to compare the efficacy and adverse effects of the two most commonly used antimetabolites - mycophenolate mofetil and azathioprine. Additionally, considering the fact that ethnicity may have an 
impact on the effect of drugs, we made an attempt to analyse the population of our patients in relation to other nations.

In the majority of published studies dealing with antiinflammatory treatment of ocular inflammations, patients come from various ethnic groups and races, for example Daniel et al. [22] reported 60.4\% - Caucasian group, 25\% - black race, Pasadhika et al. [23] 76.2\% - white race and $19 \%$ black race, Galor et al. [24] 58\% - white race and $40 \%$ black race.

All the patients recruited at our Department belong to white race and are Slavs, which could affect the effectiveness of the therapy. The impact of the ethnic origin on the course of therapy was emphasized by Cuchacovich et al. [25] who studied the population of Chilean patients diagnosed as having non-infectious inflammatory eye diseases and treated with MMF. The observation showed an increased incidence of adverse reactions among Chilean uveitis patients treated with MMF.

In our study, side effects occurred more commonly in patients taking MMF than in the AZA group. The severity of side effects was higher in the second group and more often led to discontinuation of AZA therapy. These results are comparable with data obtained by Galor et al [24]. Gastrointestinal upset was the leading symptom reported in patients treated with MMF, the effect was similar to that obtained by Daniel et al. [22], Galor et al. [24] and Teoh et al. [26].

In our opinion, the strength of our study is the uniformity of the diagnosis - only patients with uveitis, and not broadly understood inflammatory disease within the structures of the eyeball and eye orbit, were qualified for the project. In both our study groups the most common reason for the introduction of IS therapy was the failure to obtain a satisfactory outcome during systemic administration of antiinflammatory steroids. According to the guidelines, an IS drug is intended to facilitate the reduction of the daily dose of prednisone to a level not exceeding $10 \mathrm{mg}$.

Comparing the groups studied by us, steroid-sparing effectiveness was obtained significantly more commonly in the MMF group of patients than in the patients treated with AZA.

The results do not differ significantly from those obtained by other authors. In published available data- in case of treatment with MMF, the possibility of reducing the prednisone dose to $\leq 10 \mathrm{mg}$ with the suppression of uveitis was obtained in $83.3 \%$ [27], 84.6\% [26], and up to $86 \%$ of patients [22]. However, the percentage of subjects who achieved therapeutic success with a reduced dose of prednisone to $10 \mathrm{mg}$ ranged from $32 \%$ [23] to 58\% [22] among patients receiving MMF for half a year. Pasadhika et al. [23] noticed that AZA is particularly effective in the treatment of inflammation of the intermediate uveitis. Data obtained in our study confirm the efficacy of AZA in the inflammation of the intermediate tract (62.5\%). Joshi et al. [28] achieved control in a similar proportion of patients using AZA as an initial agent or after discontinuation of other agents.

Non-infectious uveitis is a heterogeneous group of diseases in which the common denominator is the presence of inflammation within the structure of the eye. Glucocorticoids are still the basic therapy. In the case of glucocorticoids treatment failure - immunosuppressive drugs are given. As the alternative - biologic response modifiers (BRM) can be used.
Biological therapy involves the introduction into the body of substances that are intended to bind to the specific molecules responsible for causing an inflammatory reaction. These drugs are being more frequently used in non-infectious uveitis treatment, especially in United States and United Kingdom [29-39].

In Poland, only adalimumab is registered for the treatment of chronic non-infectious uveitis. In 2018, Experts of the Polish Ophthalmological Society published recommendations on the use of adalimumab in two age groups of patients with uveitis. The first one - adult patients with intermediate uveitis, posterior uveitis and panuveitis who have shown insufficient response or intolerance to conventional therapy or where conventional therapy is ineffective. The second one - treatment of chronic non-infectious anterior uveitis in children and adolescents who have shown insufficient response or intolerance to conventional therapy or where conventional therapy is ineffective [40]. Previously, the only real chance for treatment with these substances was systemic disease therapy as part of the National Health Fund drug programs, in which uveitis is one of the manifestations of the disease. This has been a fundamental limitation in the possibility of using biological medicines in patients with diagnosed uveitis in the Polish population, due to the significant cost of the therapy and possible need for using off-label medicine.

On September 1, 2019 the National Health Fund approved a drug program (B.105) under which adalimumab treatment could be applied in patients with non-infectious uveitis who meet certain criteria [41]. Inclusion of adalimumab in the Drug Program created a chance to treat patients whose standard treatment was unsuccessful. In addition, as presented above, in a large proportion of patients, despite careful interdisciplinary analysis, the direct cause of uveitis cannot be found, which further complicates the use of biological treatment. Therefore, it is reasonable to conduct research that allows more reliable assessment of standard therapy.

\section{CONCLUSIONS}

Although uveitis is classified as a rare disease (38 cases per 100,000 population), it is the fourth most common cause of blindness among working people in developed countries.

In conclusion, it is noteworthy that a long-term assessment of the treatment and the course of uveitis is based on the monitoring of changes in the ability to see; before the treatment, the visual acuity did not differ statistically in patients from both assessed groups. The comparison of the data obtained after therapy revealed that over one quarter of eyes in which improvement of visual acuity was achieved belonged to the group of patients receiving mycophenolate mofetil. This result may be considered a promising therapeutic effect determined in Polish patients treated for non-infectious uveitis.

Simultaneously, we are aware of some limitations of our first study on effectiveness of applied antimetabolites. The retrospective approach is one limitation of this research. The criteria for initiating therapy with either mycophenolate mofetil or azathioprine were not strictly defined. In the early years, the financial aspect was quite important during the process of making therapeutic decisions. The second 
limitation was the difference in the duration of the disease before drug administration. Patients from Group 2 had a longer history of the disease. The third negative aspect is a relatively low number of patients assessed. In turn, the strength of this study is the uniformity of the diagnosis, as the participants qualified for the study suffered only from uveitis, and not from the broadly understood inflammatory disease within the structures of the eyeball and orbit.

Rresults of our study showing better therapeutic efficacy when mycophenolate mofetil was applied seem to define a promising approach for treatment of non-infectious posterior uveitis and panuveitis, except for intermediate uveitis in which azathioprine is preferred. In the first study, there were different duration of the disease before drug administration and limited number of patients assessed, thus it is desirable to examine more Polish patients treated with the antimetabolites.

\section{REFERENCES}

1. Rothova A, Suttorp-van Schulten MS, Frits Treffers W, Kijlstra A. Causes and frequency of blindness in patients with intraocular inflammatory disease. Br J Ophthalmol. 1996; 80: 332-336.

2. Murray PI, Rauz S. The eye and inflammatory rheumatic diseases: the eye and rheumatoid arthritis, ankylosing spondylitis, psoriatic arthritis. Best Pract Res Clin Rheumatol. 2016: 30: 802-825.

3. Read RW, Holland GN, Rao NA, Tabbara KF, Ohno S, ArellanesGarcia L, et al. Revised diagnostic criteria for Vogt-Koyanagi-Harada disease: report of an international committee on nomenclature. Am J Ophthalmol. 2001; 131: 647-652.

4. Mendes Lavezzo M, Sakata VM, Morita C, Rodriguez EE, Abdallah SF, Silva F, et al. Vogt-Koyanagi-Harada disease: review of a rare autoimmune disease targeting antigens of melanocytes. Orphanet J Rare Dis. 2016; 11: 29.

5. Silpa-archa S, Lee JJ, Foster CS. Ocular manifestations in systemic lupus erythematosus. Br J Ophthalmol. 2016 Jan; 100(1): 135-41.

6. Pasadhika S, Rosenbaum JT. Ocular Sarcoidosis. Clin Chest Med. 2015 Dec; 36(4): 669-83.

7. Jamilloux Y, Kodjikian L, Broussolle C, Sève P. Sarcoidosis and uveitis. Autoimmun Rev. 2014 Aug; 13(8): 840-9. doi: 10.1016/j. autrev.2014.04.001. Epub 2014 Apr 3.

8. Lim LL, Scarborough JD, Thorne JE, Graham E, Kempen JH, Mackensen F, Nguyen QD, Prabriputaloong T, Read RW, Suhler EB, Schwartz JM, Smith JR. Uveitis in patients with autoimmune hepatitis. Am J Ophthalmol. 2009 Feb; 147(2): 332-338.

9. Olsen TG, Frederiksen J. The association between multiple sclerosis and uveitis. Surv Ophthalmol. 2017 Jan - Feb; 62(1): 89-95. doi: 10.1016/j. survophthal.2016.07.002. Epub 2016 Aug 1.

10. Messenger W, Hildebrandt L, Mackensen F, Suhler E, Becker M, Rosenbaum JT. Characterization of uveitis in association with multiple sclerosis. Br J Ophthalmol. 2015 Feb; 99(2): 205-9.

11. Suttorp-Schulten MS, Rothova A. The possible impact of uveitis in blindness: a literature survey. Br J Ophthalmol. 1996: 80: 844-848.

12. Biziorek B, Mackiewicz J, Zagórski Z, Krwawicz L, Haszcz D. Etiology of uveitis in rural and urban areas of mid-eastern Poland. Ann Agric Environ Med. 2001; 8(2): 241-243.

13. Kwon JW, Sim Y, Jee D. Association between intermediate uveitis and toxocariasis in the Korean population. Medicine. 2017; 96: e5829.

14. Zibaei M, Alemi M, Cardillo NM, Derafshi H, Miahipour A, Bahadory S, Zarei M. Human toxocariasis seroprevalence among patients with uveitis in Alborz Province, Iran. Ann Agric Environ Med. 2019; 26(1): 154-158.

15. Raizman M. Corticosteroid therapy of eye disease. Fifty years later. Arch Ophthalmol. 1996; 114: 1000-1.

16. Lustig J, Cunningham ET, Jr. Use of immunosuppressive agents in uveitis. Curr Opin Ophthalmol. 2003; 14: 399-412.

17. Jabs DA, Rosenbaum J, Yocum D, Holland GN, Jaffe GJ, Louie JS, et al. Guidelines for the Use of Immunosuppresive Drugs in Patients with Ocular Inflammatory Disorders: recommendations of an Expert panel. Am J Ophthalmol. 2000; 130: 492-513.
18. Allison AC. Mechanisms of action of mycophenolate mofetil. Lupus 2005; 14: S2-S8.

19. Okada AA. Immunomodulatory therapy for ocular inflammatory disease: a basic manual and review of the literature. Ocul Immunol Inflamm. 2005; 13: 335-51.

20. Radzikowska E. Pneumonia in immunocompramised patients general clinical view. Pneumonol Alergol Pol. 2010; 78, 3: 236-243.

21. Jabs DA, Nussenblatt RB, Rosenbaum JT. Standardization of Uveitis Nomenclature (SUN) Working Group. Standardization of uveitis nomenclature for reporting clinical data. Results of the First International Workshop. Am J Ophthalmol. 2005; 140: 509-516.

22. Daniel E, Thorne JE, Newcomb CW, Pujari SS, Kaçmaz RO, LevyClarke GA, et al. Mycophenolate mofetil for ocular inflammation. Am J Ophthalmol. 2010; 149: 423-32.

23. Pasadhika S, Kempen JH, Newcomb CW, Liesegang TL, Pujari SS, Rosenbaum JT, et al. Azathioprine for ocular inflammatory diseases. Am J Ophthalmol. 2009; 148: 500-509.

24. Galor A, Jabs DA, Leder HA, Kedhar SR, Dunn JP, Peters GB, et al. Comparison of antimetabolite drugs as corticosteroid-sparing therapy for noninfectious ocular inflammation. Ophthalmology 2008; 115: $1826-32$.

25. Cuchacovich M, Solanes F, Perez C, Verdaguer JI, Verdaguer J, Castiglione E, Carpentier C, et al. Mycophenolate Mofetil Therapy in Refractory Inflammatory Eye Disease. J Ocul Pharmacol Ther. 2016; 32: 55-61.

26. Teoh S, Hogan A, Dick A, Lee R. Mycophenolate mofetil for the Treatment of Uveitis. Am J Ophthalmol. 2008; 146: 752-760.

27. Lau CH, Comer M, Lightman S: Long-term efficacy of mycophenolate mofetil in the control of severe intraocular inflammation. Clin Exp Ophthalmol. 2003; 31: 487-91.

28. Joshi L, Talat L, Yaganti S, Sandhu S, Taylor S, Wakefield D, et al. Outcomes of Changing Immunosuppressive Therapy after Treatment Failure in Patients with Noninfectious Uveitis. Ophthalmol. 2014; 121(5): 1119-1124.

29. Levy-Clarke G, Jabs DA, Read RW, Rosenbaum JT, Vitale A, Van Gelder RN. Expert panel recommendations for the use of anti-tumor necrosis factor biologic agents in patients with ocular inflammatory disorders. Ophthalmology. 2014; 121(3): 785-796. e3

30. Rudwaleit M, Rødevand E, Holck P, Vanhoof J, Kron M, Kary S, et al. Adalimumab effectively reduces the rate of anterior uveitis flares in patients with active ankylosing spondylitis: results of a prospective open-label study. Ann Rheum Dis. 2009; 68(5): 696-701.

31. Kruh JN, Yang P, Suelves AM, Foster CS. Infliximab for the treatment of refractory noninfectious uveitis: A study of 88 patients with longterm follow-up. Ophthalmology. 2014; 121(1): 358-364.

32. Jaffe GJ, Dick AD, Brézin AP, et al. Adalimumab in Patients with Active Noninfectious Uveitis. N Engl J Med. 2016; 375(10): 932-943.

33. Dobner BC, Max R, Becker MD, Heinz C, Veltrup I, Heiligenhaus A, et al. A three-centre experience with adalimumab for the treatment of non-infectious uveitis. Br J Ophthalmol. 2013; 97(2): 134-138.

34. Heiligenhaus A, Thurau S, Wildner G, Grajewski RS, Wildner G. Antiinflammatory treatment of uveitis with biologicals: new treatment options that reflect pathogenetic knowledge of the disease. Graefes Arch Clin Exp Ophthalmol. 2010: 248: 1531-1551.

35. Yazgan S, Celik U, Işık M, Yeşil NK, Baki AE, Şahin H, et al. Efficacy of golimumab on recurrent uveitis in HLA-B27-positive ankylosing spondylitis. Int Ophthalmol. 2017 Feb; 37(1): 139-145.

36. Silpa-Archa S, Oray M, Preble JM, Foster CS: Outcome of tocilizumab treatment in refractory ocular inflammatory diseases. Acta Ophthalmol. 2016; 94(6): e400-406.

37. Emmi G, Talarico R, Lopalco G, Cimaz R, Cantini F, Viapiana O, et al. Efficacy and safety profile of anti-interleukin-1 treatment in Behçet's disease: a multicenter retrospective study. Clin Rheumatol. 2016; 35(5): 1281-1286.

38. Tugal-Tutkun I, Pavesio C, De Cordoue A, Bernard-Poenaru O, Gül A. Use of Gevokizumab in Patients with Behçet's Disease Uveitis: An International, Randomized, Double-Masked, Placebo-Controlled Study and Open-Label Extension Study. Ocul Immunol Inflamm. 2018; 25: 1-11.

39. Heiligenhaus A, Miserocchi E, Heinz C, Gerloni V, Kotaniemi K. Treatment of severe uveitis associated with juvenile idiopathic arthritis with anti-CD20 monoclonal antibody (rituximab). Rheumatology (Oxford). 2011; 50(8): 1390-1394.

40. https://pto.com.pl/storage/guidelines/36/c382c42a5f07be6abc075c60de4892ec.pdf

41. http://dziennikmz.mz.gov.pl/api/DUM_MZ/2019/65/journal/5588 